\title{
Spatial Heterogeneity and Imperfect Mixing in Chemical Reactions: Visualization of Density-Driven Pattern Formation
}

\author{
Sabrina G. Sobel, ${ }^{1}$ Harold M. Hastings, ${ }^{2}$ and Matthew Testa ${ }^{2}$ \\ ${ }^{1}$ Department of Chemistry, Hofstra University, Hempstead, NY 11549-1510, USA \\ ${ }^{2}$ Department of Physics and Astronomy, Hofstra University, Hempstead, NY 11549-1510, USA \\ Correspondence should be addressed to Sabrina G. Sobel, sabrina.sobel@hofstra.edu
}

Received 16 February 2009; Accepted 3 April 2009

Recommended by Q. Xu

Imperfect mixing is a concern in industrial processes, everyday processes (mixing paint, bread machines), and in understanding salt water-fresh water mixing in ecosystems. The effects of imperfect mixing become evident in the unstirred ferroin-catalyzed Belousov-Zhabotinsky reaction, the prototype for chemical pattern formation. Over time, waves of oxidation (high ferriin concentration, blue) propagate into a background of low ferriin concentration (red); their structure reflects in part the history of mixing in the reaction vessel. However, it may be difficult to separate mixing effects from reaction effects. We describe a simpler model system for visualizing density-driven pattern formation in an essentially unmixed chemical system: the reaction of pale yellow $\mathrm{Fe}^{3+}$ with colorless $\mathrm{SCN}^{-}$to form the blood-red $\mathrm{Fe}(\mathrm{SCN})^{2+}$ complex ion in aqueous solution. Careful addition of one drop of $\mathrm{Fe}\left(\mathrm{NO}_{3}\right)_{3}$ to KSCN yields striped patterns after several minutes. The patterns appear reminiscent of Rayleigh-Taylor instabilities and convection rolls, arguing that pattern formation is caused by density-driven mixing.

Copyright (c) 2009 Sabrina G. Sobel et al. This is an open access article distributed under the Creative Commons Attribution License, which permits unrestricted use, distribution, and reproduction in any medium, provided the original work is properly cited.

\section{Introduction}

Good mixing is a major challenge in many areas of chemistry and chemical engineering, ranging from continuously stirred tank reactors (CSTRs) [1] to industrial level processes [2], where imperfect mixing can adversely affect product quality and yield. Many of us are familiar with mixing problems in making bread, stirring paint, mixing gasoline and air in internal combustion engines, and mixing driven by density differences (salt water-fresh water convection) but even mixing of ordinary liquids can pose challenges. (The density of pure water is $1000 \mathrm{~kg} / \mathrm{m}^{3}$. Ocean water is more dense because of the salt in it. Density of ocean water at the sea surface is about $1027 \mathrm{~kg} / \mathrm{m}^{3}$. There are two main factors that make ocean water more or less dense than about $1027 \mathrm{~kg} / \mathrm{m}^{3}$. From http://www.csgnetwork.com/h2odenscalc.html we derived Table 1 (accessed 1/2009)).

The effects of imperfect mixing are perhaps most dramatically evident in excitable chemical systems $[1,3]$ such as the $\mathrm{BZ}$ reaction [4-9] and the chlorite-iodide reaction [10-12]. An excitable chemical reaction is a reaction in which suitable small perturbations from steady state generate large excursions (excitations) before the system returns to steady state. Many excitable reactions display auto-oscillatory behavior in which sustained oscillations about an unstable steady state are observed. The effects of imperfect mixing can be seen readily in the unstirred, ferroin-catalyzed BeloushovZhabotinsky (BZ) reaction in a Petri disk, a quasi-twodimensional (2D) system [3-8]. The catalyst in this reaction, ferroin/ferriin, also serves as an indicator; blue/oxidized at high [ferriin] and red/reduced at low [ferriin]. After an induction period of several minutes, one sees the "spontaneous" formation of target patterns of blue/oxidized rings moving outward from oscillatory (red/blue) centers into a red/reduced background.

Menzinger and Dutt [1] first described the effects of imperfect mixing upon reactions involving excitable media. We have explicitly demonstrated nonrandom spatiotemporal order in target formation in the unstirred BZ reaction, with targets preferentially occurring in excitable regions near existing targets, but too far from pre-existing targets to be generated by wave propagation or chemical diffusion [3]. 

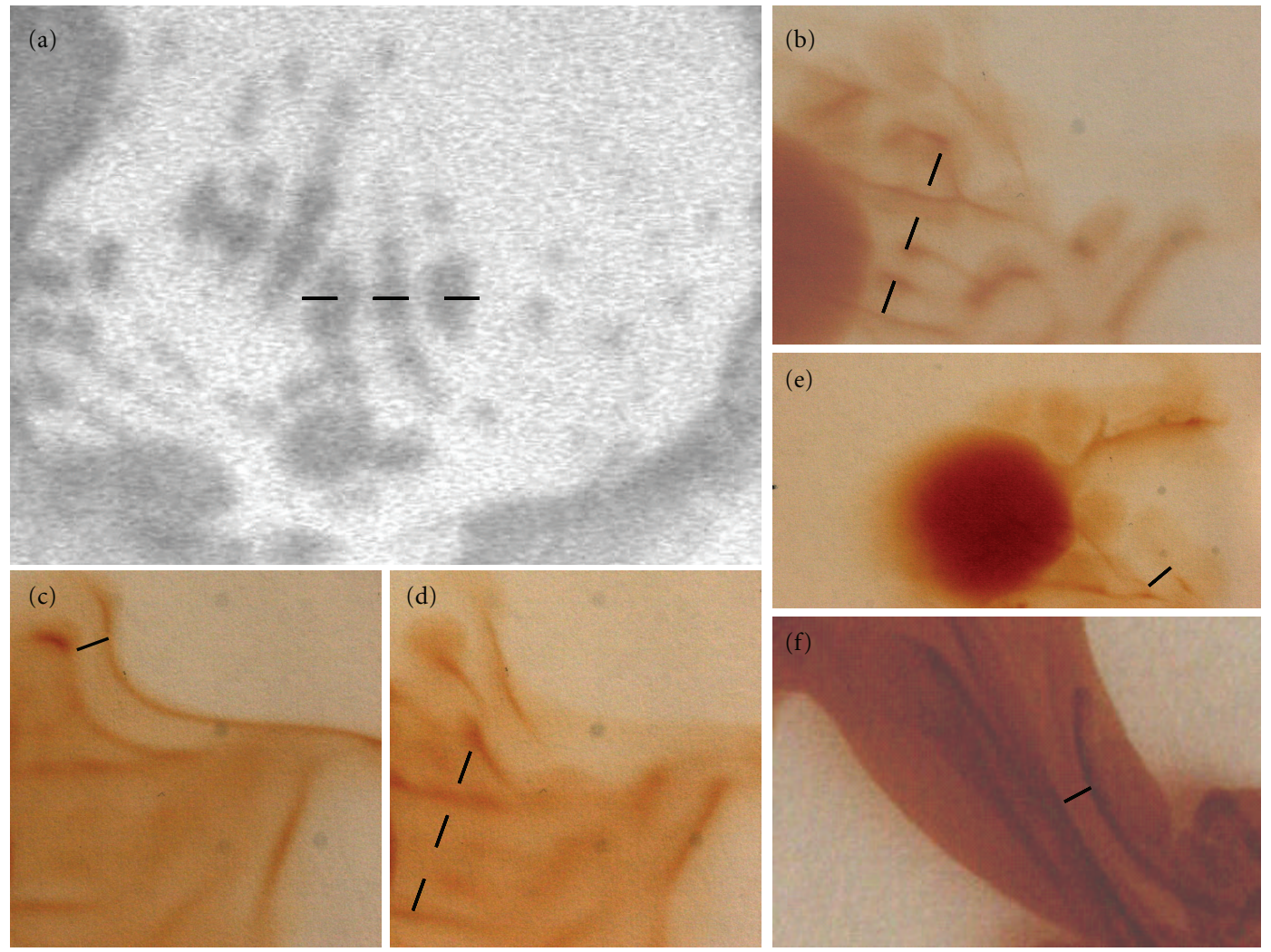

FIGURE 1: (a) Excitable BZ reaction medium, stirred after initial pattern formation, $2.1 \mathrm{~mm}$ depth. From [9, Figure 2c]. Scale bar: alternating $2.1 \mathrm{~mm}$ lines and spaces. (b) Chemical model: $\mathrm{Fe}^{3+}$ and $S C \mathrm{~N}^{-}, 2 \mathrm{~mm}$ depth. Scale bar: alternating $2 \mathrm{~mm}$ lines and spaces. Striped patterns formed outside the visible droplet boundary (lower left of figure). The color corresponds to the concentration of FeSCN ${ }^{2+}$. Resolution $-30 \mu \mathrm{m}$ in photos (b) through (f). Time: about two minutes after addition of one drop of Fe ${ }^{3+}$ solution. (c), (d) Evolution of panel (b). Same experimental run, 45 and 30 second earlier. Note pattern initiation $2 \mathrm{~mm}$ behind visible wavefront (of diffusion wave) and effect of diffusion in blurring visible boundaries as concentration gradients are reduced. (e) Same model, but $3 \mathrm{~mm}$ depth, early stage of pattern formation. Note visible droplet boundary in center of figure, and larger scale that corresponds to greater depth as in (b) through (d). (f) Same model, $1 \mathrm{~mm}$ depth, early stage of pattern formation. Scale bar: $1 \mathrm{~mm}$. Note fine structure $(\ll 1 \mathrm{~mm})$ transverse to a spatial filament of the reaction, and initial excitation of $1 \mathrm{~mm}$ scale.

We explained the observed spatiotemporal order in terms of mixing heterogeneities in preparing the $2 \mathrm{D}$ reaction medium likely because of the sensitivity of the dynamics to very small perturbations at the onset of oscillation [13, 14]. In conditions of almost complete mixing, striped patterns reminiscent of convection rolls with spacing approximately equal to the depth of the medium were often observed (Figure 1, panel (a)). Here we describe an even simpler model system which displays analogous pattern formation driven by density differences, c.f. [15-17].

\section{Pattern Visualization with $\mathrm{Fe}(\mathrm{SCN})^{2+}$}

The present experiment arose from trying to understand how slight density differences might drive this pattern formation. Since BZ dynamics are relatively complex, we sought a simpler, minimal example to better visualize and understand the effects of mixing heterogeneity. We used a simple chemical reaction, in which two nearly colorless solutions, one containing $\mathrm{Fe}^{3+}$ (pale yellow) and the second containing $\mathrm{SCN}^{-}$(clear), are mixed, producing a deep reddish color
TABLe 1: Densities of salt water and pure water at various temperatures

\begin{tabular}{lcc}
\hline Temp $\left({ }^{\circ} \mathrm{C}\right)$ & Ionic strength & Calc. density $\left(\mathrm{g} / \mathrm{cm}^{3}\right)$ \\
\hline 23 & 3500 & 1.000215 \\
& 0 & 0.997568 \\
26 & 0 & 0.996814 \\
20 & 0 & 0.998234 \\
30 & 0 & 0.995678 \\
\hline
\end{tabular}

where $\mathrm{Fe}^{3+}$ and $\mathrm{SCN}^{-}$combine to form the complex ion $\mathrm{FeSCN}^{2+}$ in an essentially irreversible reaction. We made a simple modification-instead of mixing the two solutions, we added a small drop $(<1 \mu \mathrm{L})$ of $0.1 \mathrm{M} \mathrm{Fe}^{3+}$ (as $\left.\mathrm{Fe}\left(\mathrm{NO}_{3}\right)_{3}\right)$ to a 1-4 mm deep solution of $0.02 \mathrm{M} \mathrm{SCN}^{-}$(as KSCN) optionally containing a very small amount of surfactant (which had no apparent effect). The deep reddish color where $\mathrm{Fe}^{3+}$ and $\mathrm{SCN}^{-}$combine to form $\mathrm{FeSCN}{ }^{2+}$ clearly labels interfaces between $\mathrm{Fe}^{3+}$-dominated and $\mathrm{SCN}^{-}$-dominated regions. We found patterns reminiscent of convective rolls 
with spacing equal to the depth of the corresponding reaction medium (Figure 1, panels (b)-(e), below, similar to patterns seen under conditions of almost complete mixing in the $\mathrm{BZ}$ reaction, panel (a)). Note that the $\mathrm{Fe}\left(\mathrm{NO}_{3}\right)_{3}$ solution is denser than the KSCN solution: 1.010(4) versus $1.001(2) \mathrm{g} / \mathrm{mL}$.

The resulting patterns demonstrate behavior expected in the Rayleigh-Taylor instability $[18,19]$, which occurs when a heavier fluid (here the $\mathrm{Fe}\left(\mathrm{NO}_{3}\right)_{3}$ solution) is placed on top of a lighter fluid (here the KSCN solution). Gravitational forces drive the heavier solution downward through the lighter solution in an unstable, turbulent manner, until concentration (and thus density) differences are destroyed by diffusion. In our model system, one sees initial small scale instabilities (panel (f)) which are gradually destroyed by diffusion as longer length scales are excited, with one added twist - selection of a length scale corresponding to the depth of the reaction medium after a time delay a few multiples of the time it takes a drop to fall the depth of the solution. From Stokes law, the net force on a small drop depends upon its volume, velocity, and density difference $\left(\rho_{1}-\rho_{2}\right)$. In our model a drop of radius $50 \mu \mathrm{m}$ rapidly reaches terminal velocity of $\sim 50 \mu \mathrm{m} / \mathrm{s}$, making the natural time scale (time to fall $2 \mathrm{~mm}) \sim 2 \mathrm{~mm} /(50 \mu \mathrm{m} / \mathrm{s})=40$ second, consistent with the formation of relatively stable patterns over $\sim 3$ minutes ( $\sim 4$ times this natural time scale).

\section{Conclusion}

This simple minimal model, in a nonexcitable system, demonstrates excitation of the most unstable mode (the only one that survives over minutes). It visually demonstrates the persistence of spatial heterogeneities in liquids. The problem of mixing is important in real-world situations often encountered by chemical engineers (industrial chemical reactions), process/flow engineers, and even fields such as hydrology and geoscience-temperature and salinity differences can cause density differences in water similar in magnitude to the density differences in this experimental model. This simple model provides a mechanism to experimentally visualize and study the patterns formed by density differences.

\section{Acknowledgments}

This material is based upon work supported by the US Department of Energy under Award no. DE-FG0208ER64623 for the Hofstra University Center for Condensed Matter Research and by the US National Science Foundation Grant CHE-0515691. Neither the United States Government nor any agency thereof, nor any of their employees, makes any warranty, expressed or implied, or assumes any legal liability or responsibility for the accuracy, completeness, or usefulness of any information, apparatus, product, or process disclosed, or represents that its use would not infringe privately owned rights. Reference herein to any specific commercial product, process, or service by trade name, trademark, manufacturer, or otherwise does not necessarily constitute or imply its endorsement, recommendation, or favoring by the United States Government or any agency thereof. The views and opinions of authors expressed herein do not necessarily state or reflect those of the United States Government or any agency thereof.

\section{References}

[1] M. Menzinger and A. K. Dutt, "The myth of the well-stirred CSTR in chemical instability experiments: the chlorite/iodide reaction," Journal of Physical Chemistry, vol. 94, no. 11, pp. 4510-4514, 1990.

[2] E. L. Paul, V. Atiemo-Obeng, and S. M. Kresta, Eds., Handbook of Industrial Mixing: Science and Practice, Wiley-Interscience, Hoboken, NJ, USA, 2004.

[3] H. M. Hastings, S. G. Sobel, A. Lemus, et al., "Spatiotemporal clustering and temporal order in the excitable BZ reaction," Journal of Chemical Physics, vol. 123, no. 6, Article ID 064502, 6 pages, 2005.

[4] B. P. Belousov, "A periodic reaction and its mechanism," in Sbornick Referatov po Radiatsionni Meditsine, pp. 145-160, Medgiz, Moscow, Russia, 1958.

[5] A. M. Zhabotinsky, "Periodic processes of the oxidation of malonic acid in solution (study of the kinetics of Belousov's reaction)," Biofizika, vol. 9, p. 306, 1964.

[6] R. J. Field, E. Körös, and R. M. Noyes, "Oscillations in chemical systems. II. Thorough analysis of temporal oscillation in the bromate-cerium-malonic acid system," Journal of the American Chemical Society, vol. 94, no. 25, pp. 8649-8664, 1972.

[7] R. J. Field and R. M. Noyes, "Oscillations in chemical systems. V. Quantitative explanation of band migration in the Belousov-Zhabotinskii reaction," Journal of the American Chemical Society, vol. 96, no. 7, pp. 2001-2006, 1974.

[8] B. Z. Shakashiri, Chemical Demonstrations, Vol. 2, University of Wisconsin Press, Madison, Wis, USA, 1985.

[9] S. K. Scott, Oscillations, Waves, and Chaos in Chemical Kinetics, Oxford Chemistry Primers no. 18, Oxford University Press, New York, NY, USA, 1994.

[10] C. E. Dateo, M. Orban, P. De Kepper, and I. R. Epstein, "Systematic design of chemical oscillators. 5. Bistability and oscillations in the autocatalytic chlorite-iodide reaction in a stirred-flow reactor," Journal of the American Chemical Society, vol. 104, no. 2, pp. 504-509, 1982.

[11] M. Orban, C. Dateo, P. De Kepper, and I. R. Epstein, "Systematic design of chemical oscillators. 11. Chlorite oscillators: new experimental examples, tristability, and preliminary classification," Journal of the American Chemical Society, vol. 104, no. 22, pp. 5911-5918, 1982.

[12] O. Citri and I. R. Epstein, "Systematic design of chemical oscillators. 42. Dynamic behavior in the chlorite-iodide reaction: a simplified mechanism," Journal of Physical Chemistry, vol. 91, no. 23, pp. 6034-6040, 1987.

[13] H. M. Hastings, R. J. Field, and S. G. Sobel, "Microscopic fluctuations and pattern formation in a supercritical oscillatory chemical system," Journal of Chemical Physics, vol. 119, no. 6, pp. 3291-3296, 2003.

[14] H. M. Hastings, S. G. Sobel, R. J. Field, et al., "Bromide control, bifurcation and activation in the Belousov-Zhabotinsky reaction," Journal of Physical Chemistry A, vol. 112, no. 21, pp. 4715-4718, 2008. 
[15] Y. Wu, D. A. Vasquez, B. F. Edwards, and J. W. Wilder, "Convective chemical-wave propagation in the BelousovZhabotinsky reaction," Physical Review E, vol. 51, no. 2, pp. 1119-1127, 1995.

[16] D. Zhang, W. R. Peltier, and R. L. Armstrong, "Buoyant convection in the Belousov-Zhabotinsky reaction. I. Thermally driven convection and distortion of chemical waves," The Journal of Chemical Physics, vol. 103, no. 10, pp. 4069-4077, 1995.

[17] D. Zhang, W. R. Peltier, and R. L. Armstrong, "Buoyant convection in the Belousov-Zhabotinsky reaction. II. Chemically driven convection and instability of the wave structure," The Journal of Chemical Physics, vol. 103, no. 10, pp. 4078-4089, 1995.

[18] A. W. Cook and D. Youngs, "Rayleigh-Taylor instability and mixing," Scholarpedia, vol. 4, no. 2, p. 6092, 2009.

[19] M. J. Andrews and D. B. Spalding, "A simple experiment to investigate two-dimensional mixing by Rayleigh-Taylor instability," Physics of Fluids A, vol. 2, no. 6, pp. 922-927, 1990. 


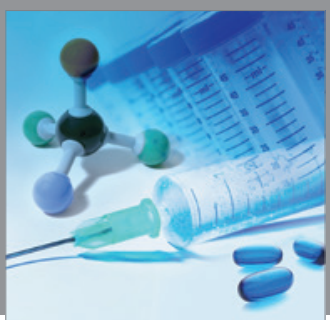

International Journal of

Medicinal Chemistry

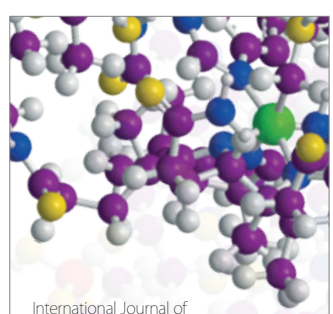

Carbohydrate Chemistry

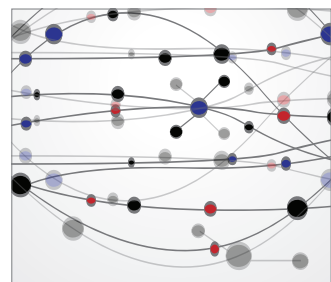

The Scientific World Journal
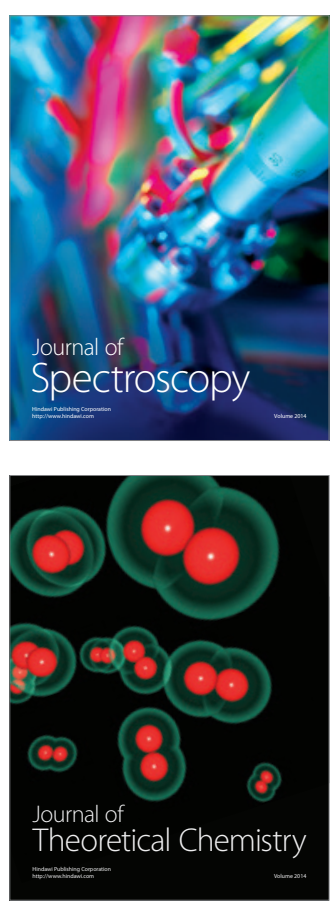
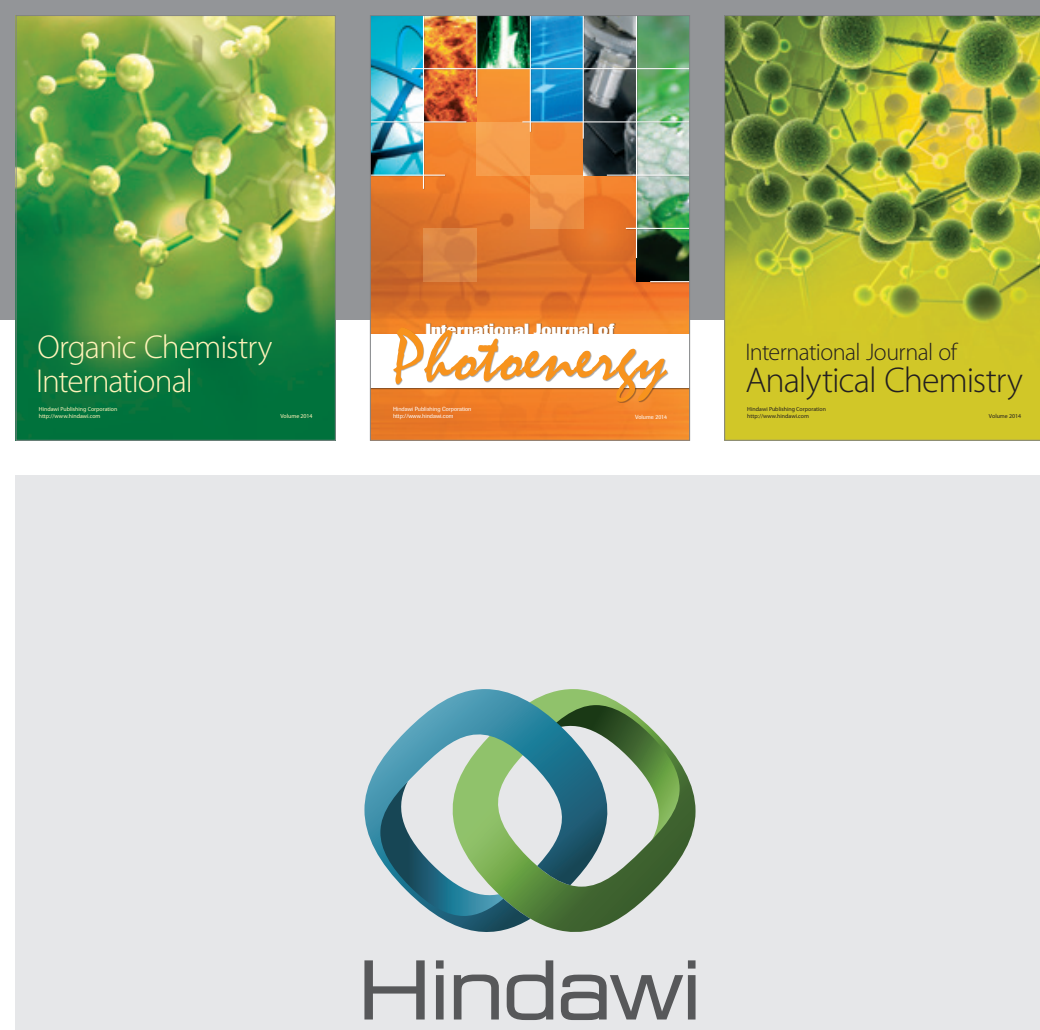

Submit your manuscripts at

http://www.hindawi.com
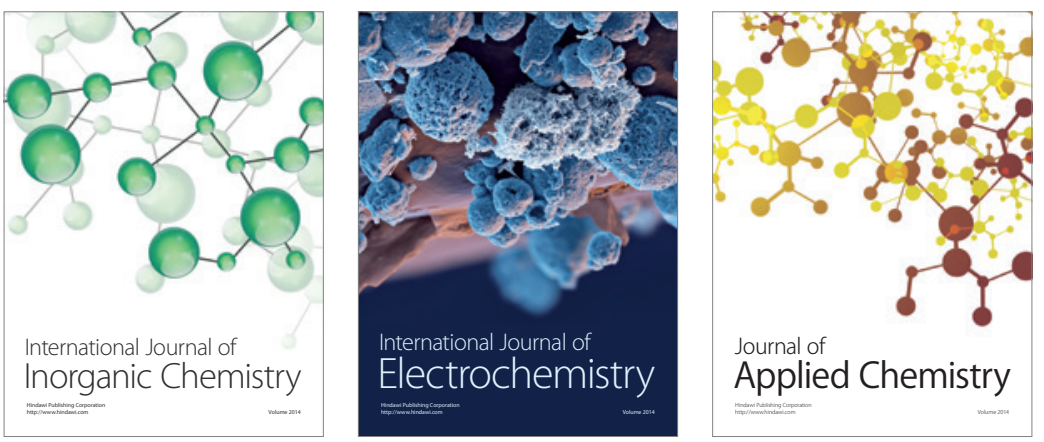

Journal of

Applied Chemistry
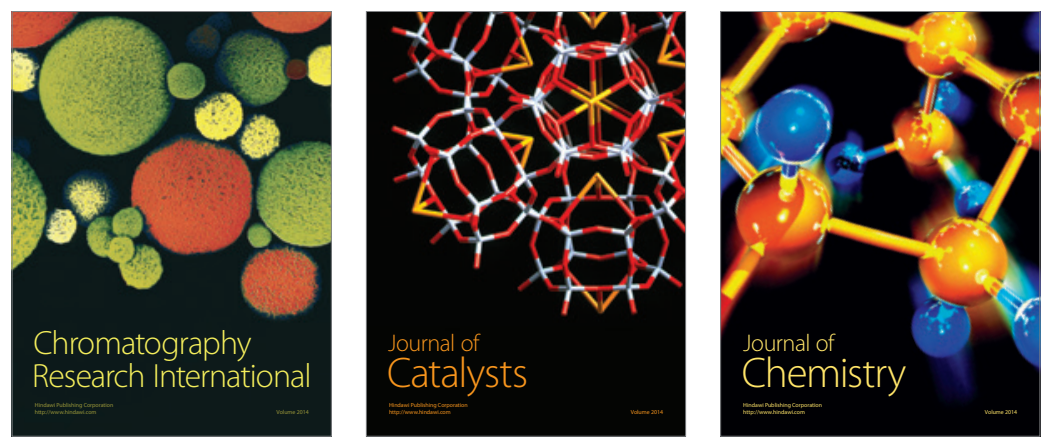
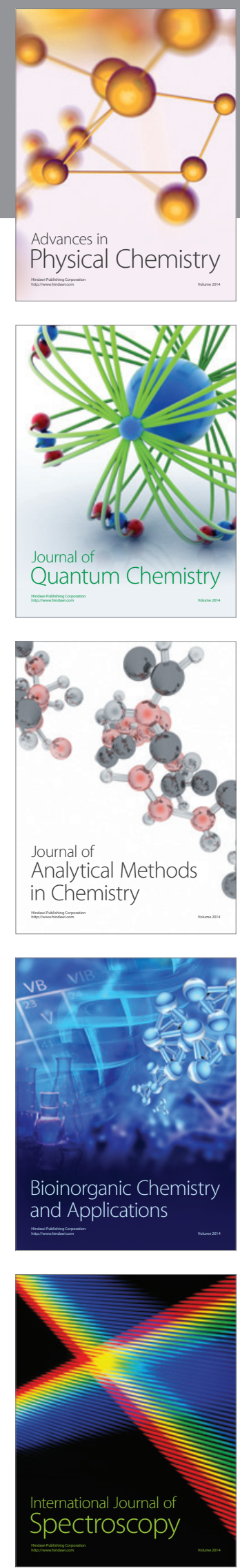